\title{
Spleen transplant holds promise as disease cure
}

Genetic diseases caused by mutations in single genes have long been the darling of gene therapy efforts. In a twist on this theme, a team from the Weizmann Institute of Science (Rehovot, Israel) has shown that embryonic spleen transplantation can correct at least one monogenic disease in mice, a proof of concept that may be applicable to humans.

Yair Reisner and his colleagues, writing in the Proceedings of the National Academy of Sciences, describe their bid to cure hemophilia A in mice by means of fetal pig spleen tissue transplantation. Hemophilia $\mathrm{A}$ is a genetic disease arising from mutation in the gene for the clotting protein factor VIII (FVIII), which renders the protein unable to function and results in uncontrollable hemorrhage from injury or normal wear and tear.

Previous efforts at spleen transplantation have been plagued by graft-versus-host disease, a condition seen in bone marrow and other transplants in which residual donor $\mathrm{T}$ cells in the graft attack the tissue recipient. Reisner and his coworkers chose to use embryonic spleen tissue because such tissue, if derived from an early-stage fetus, is without $\mathrm{T}$ cells, and therefore unable to induce graft-versus-host disease.

The researchers harvested fetal pig spleen at embryonic day 42 , at which point no $\mathrm{T}$ cells have formed, and transplanted that tissue into SCID mice deficient in the FVIII gene. Within 2-3 months, the transplanted spleens were producing enough FVIII to completely resolve all clinical manifestations of hemophilia (such as excessive bleeding). Moreover, in a separate experiment, the researchers demonstrated that the pig spleen transplants could be tolerated by immunocompetent C57BL/6 mice with the help of immune suppressors.

Taken as a whole, these results indicate that embryonic pig spleen transplants may work in humans to treat or cure patients with genetic deficiencies, such as hemophilia, phenylketonuria, or other monogenic disorders. The main obstacle to adapting these treatments to humans may be the immunosuppressant therapy required to prevent the pig spleen tissue from being rejected in humans,

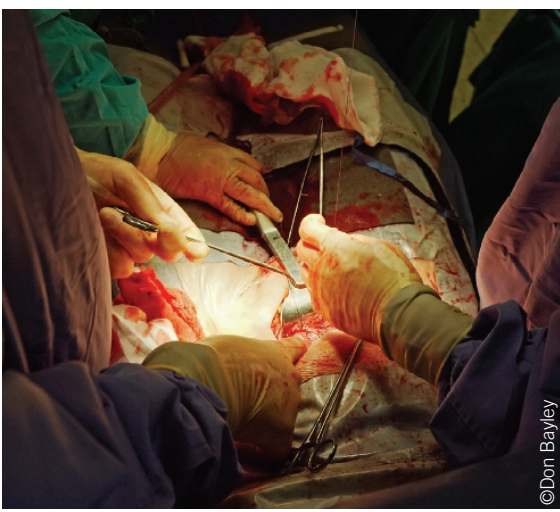

as such therapy carries its own health risks. For that reason, embryonic pig spleen transplants may be most suitable for diseases that have no other suitable treatment. Another possible, though morally ambiguous, variation on Reisner's technique would be to use spleen tissue taken from human fetuses aborted at an early stage, because such tissue is likely to present comparatively less chance of rejection from a human host.

Owen Young

\section{BLOCKTNG BRCA1 BREAST CANCER}

Inhibiting progesterone may prevent breast cancer in women with a certain genetic predisposition to the disease, according to the results of a new study in mice that could lead to the development of a new drug for breast cancer prophylaxis.

Women carrying a mutation in the tumor suppressor gene BRCA1 are up to seven times more likely to develop breast cancer than women without the mutation. Mutated BRCA1, most common in women of Ashkenazi Jewish background, also increases the risk of ovarian and other cancers, although the mechanism of BRCA1mediated cancer development is unclear.

Women with a family history of breast or ovarian cancer can undergo genetic testing, but, at present, carriers of mutated $B R C A 1$ have two less-than-ideal choices: rely on constant monitoring to try to detect the cancer at an early and curable stage, or opt for breast and ovary removal to eliminate the possibility of developing cancer in the first place. For many atrisk women, a pharmacological intervention would be a welcome alternative.

Previous epidemiological and laboratory research has suggested that progesterone has a role in driving breast cancer development. Hormone replacement therapy with estrogen and progesterone, but not estrogen alone, increases the risk of breast cancer in postmenopausal women. In addition, in cell culture, the BRCA1 protein has been shown to inhibit the action of the progesterone receptor.
In the 1 December 2006 issue of Science, Eva Lee and her colleagues at the University of California, Irvine describe work in mice that may lay the groundwork for pharmacological prevention of breast cancer. Lee's team studied nullparous mice with mutant BRCA1 and p53 (another tumor suppressor gene that is commonly mutated in breast cancer patients). These mice had the increased growth and branching of mammary tissue normally seen during pregnancy. Moreover, all of the double-mutant mice developed breast cancer by eight months of age. $0 \mathrm{n}$ the other hand, mice treated with mifepristone (RU 486), a progesterone inhibitor that is used in humans to induce abortion, remained cancer-free at 12 months of age. Mammary cells from the double-mutant mice expressed more progesterone receptors than those from controls or p53-mutant mice

Mouse and human mammary cell cultures showed that functional BRCA1 protein is required for the timely breakdown of the progesterone receptor. Inactivated BRCA1 leads to the presence of more progesterone receptors, which in turn leads to more progesterone-mediated cell proliferation, increasing the chance that a cancer-promoting mutation will occur during cell division.

Although mifepristone seemed to work wonders in mice, the fact that it acts on several hormone receptors may mean that it is not be appropriate for long-term use. Lee tells Lab Animal that her team is currently working to identify more-specific progesterone blockers. Tanja Schub 